OPEN ACCESS

Edited by:

Ovidiu Constantin Baltatu, Anhembi Morumbi University, Brazil

Reviewed by:

Hauke Busch,

University of Lübeck, Germany

David Sedmera,

Charles University, Czechia Poongodi Geetha-Loganathan, State University of New York at Oswego, United States

*Correspondence:

Sandra Rugonyi rugonyis@ohsu.edu

Specialty section:

This article was submitted to Integrative Physiology, a section of the journal Frontiers in Physiology

Received: 18 May 2017 Accepted: 14 August 2017 Published: 30 August 2017

Citation:

Midgett M, López CS, David L, Maloyan A and Rugonyi S (2017) Increased Hemodynamic Load in Early Embryonic Stages Alters Myofibril and Mitochondrial Organization in the Myocardium. Front. Physiol. 8:631. doi: 10.3389/fphys.2017.00631

\section{Increased Hemodynamic Load in Early Embryonic Stages Alters Myofibril and Mitochondrial Organization in the Myocardium}

\author{
Madeline Midgett ${ }^{1}$, Claudia S. López ${ }^{1,2}$, Larry David ${ }^{3}$, Alina Maloyan ${ }^{4}$ and \\ Sandra Rugonyi ${ }^{1 *}$ \\ ${ }^{1}$ Biomedical Engineering, Oregon Health \& Science University, Portland, OR, United States, ${ }^{2}$ Multiscale Microscopy Core, \\ OHSU Center for Spatial Systems Biomedicine, Oregon Health \& Science University, Portland, OR, United States, \\ ${ }^{3}$ Proteomics Core, Oregon Health \& Science University, Portland, OR, United States, ${ }^{4}$ Knight Cardiovascular Institute, \\ Oregon Health \& Science University, Portland, OR, United States
}

Normal blood flow is essential for proper heart formation during embryonic development, as abnormal hemodynamic load (blood pressure and shear stress) results in cardiac defects seen in congenital heart disease (CHD). However, the detrimental remodeling processes that relate altered blood flow to cardiac malformation and defects remain unclear. Heart development is a finely orchestrated process with rapid transformations that occur at the tissue, cell, and subcellular levels. Myocardial cells play an essential role in cardiac tissue maturation by aligning in the direction of stretch and increasing the number of contractile units as hemodynamic load increases throughout development. This study elucidates the early effects of altered blood flow on myofibril and mitochondrial configuration in the outflow tract myocardium in vivo. Outflow tract banding was used to increase hemodynamic load in the chicken embryo heart between Hamburger and Hamilton stages 18 and 24 ( 24 h during tubular heart stages). 3D focused ion beam scanning electron microscopy analysis determined that increased hemodynamic load induced changes in the developing myocardium, characterized by thicker myofibril bundles that were more disbursed in circumferential orientation, and mitochondria that organized in large clusters around the nucleus. Proteomic mass-spectrometry analysis quantified altered protein composition after banding that is consistent with altered myofibril thin filament assembly and function, and mitochondrial maintenance and organization. Additionally, pathway analysis of the proteomics data identified possible activation of signaling pathways in response to banding, including the renin-angiotensin system (RAS). Imaging and proteomic data combined indicate that myofibril and mitochondrial arrangement in early embryonic stages is a critical developmental process that when disturbed by altered blood flow may contribute to cardiac malformation and defects.

Keywords: cardiac development, hemodynamically-induced cardiac remodeling, outflow tract development, congenital heart disease, embryonic myocardial maturation, hemodynamic regulation of heart development 


\section{INTRODUCTION}

The heart is the first functional organ in the embryo, and starts beating by the coordinated interaction of primitive myofibril bundles and energy supplying mitochondria as soon as the early heart tube is formed (Wainrach and Sotelo, 1961). These premature organelles are not regularly organized as in the mature heart. Instead, disarrayed myofibril-like structures and mitochondria are distributed throughout the cytoplasm and lack alignment and packing in early embryonic myocytes (Smolich, 1995). Throughout normal development, myofibril and mitochondrial cell volume fractions increase, myofibril bundles align to the longitudinal axis of the myocyte (which themselves align in the direction of maximal contraction), and myofibrils together with mitochondria arrange in an orderly pattern (Fischman, 1967; Manasek, 1970; Brook et al., 1983; Rai et al., 2008). These developmental changes take place in order to ensure optimal force transfer and contraction in the heart (Raeker et al., 2014). Mature myofibrils emerge in late embryonic stages, and are characterized by an aligned and organized repeated pattern of thick (actin) and thin (myosin) filaments that are connected by sarcoplasmic-derived Z-discs (Manasek, 1970). Further, in late fetal and adult periods, myocardial mitochondria are stacked in orderly rows within a myofibrillar lattice, and contact surrounding ATP consumption sites to aid in contraction (Roberts et al., 1979; Vendelin et al., 2005).

Blood flow dynamics play a critical role in regulating early cardiac morphogenesis (Culver and Dickinson, 2010). Hemodynamic forces exerted on cardiac tissue walls trigger mechanotransduction mechanisms that lead to physical, chemical, and gene regulatory responses (Davies, 1995). Numerous studies have shown that surgically altered blood flow results in a spectrum of cardiac defects seen in human congenital heart disease (CHD; Clark and Rosenquist, 1978; Clark et al., 1989; Hogers et al., 1997, 1999; Sedmera et al., 1999; Tobita et al., 2002; Hu N. et al., 2009; Midgett and Rugonyi, 2014), however the ways in which altered blood flow triggers malformation remain unclear. Understanding the root causes of abnormal development in embryonic stages that leads to congenital heart defects is essential for future prevention and treatment.

This study specifically investigated myocardial changes in myofibril and mitochondrial organization following altered hemodynamics in the chicken embryo cardiac outflow tract. The outflow tract connects the primitive ventricle with the arterial vessel system in the early embryonic tubular heart. Outflow tract remodeling is frequently studied because the outflow tract gives rise to structures that are often involved in congenital heart defects including the aorta, pulmonary trunk, a portion of the interventricular septum, and semilunar valves. We used a wellestablished hemodynamic intervention, outflow tract banding, to alter blood flow in the chicken embryo at Hamburger and Hamilton (HH) stage 18 ( $\sim 3$ days of incubation; Hamburger

\footnotetext{
Abbreviations: CLUH, Clustered Mitochondria Homolog; FIB-SEM, Focused ion Beam Scanning Electron Microscopy; HH, Hamburger and Hamilton Development Stage; MRP, Mitochondrial Ribosomal Protein; OCT, Optical Coherence Tomography; TEM, Transmission Electron Microscopy; TMT, Tandem mass Tagging.
}

and Hamilton, 1992), in order to characterize changes in normal myofibril and mitochondrial development induced by increased hemodynamic load in vivo. Outflow tract banding increases blood pressure throughout the circulatory system (Tobita et al., 2002; Shi et al., 2013) and blood flow velocities in the constricted region of the outflow tract (Rugonyi et al., 2008; Midgett et al., 2014). Blood pressure and blood flow velocity increases strongly depended on the degree of band tightness (Shi et al., 2013; Midgett et al., 2014) and result in a wide spectrum of heart defects in the chicken embryo (Clark and Rosenquist, 1978; Clark et al., 1989; Hogers et al., 1997; Sedmera et al., 1999; Tobita et al., 2002; Midgett et al., 2017b). We used chicken embryos as a model of human heart development (which is highly conserved among vertebrate species) to allow for ease of accessibility in the egg for surgical manipulation and in vivo imaging (McQuinn et al., 2007; Rugonyi et al., 2008; Shi et al., 2013). This work elucidates how increased hemodynamic load detrimentally interferes with normal myofibril and mitochondrial arrangement, which may be fundamental in understanding the origins of CHD. Specifically, this study indicates that normal myocardial and mitochondrial reorganization is one of the initial processes affected by increased hemodynamic load. Banded samples had thicker and more randomly oriented myofibril bundles and mitochondria organized in larger clusters compared to controls. These changes were accompanied by abnormal proteomic expression, possibly implicating the renin-angiotensin system (RAS) in the early response to altered hemodynamic load by banding.

\section{METHODS}

\section{Hemodynamic Intervention}

Fertilized White Leghorn chicken eggs were incubated blunt end up at $38^{\circ} \mathrm{C}$ and $80 \%$ humidity until stage $\mathrm{HH} 18$ ( $\sim 3$ days; Hamburger and Hamilton, 1992), when the heart is a looped tubular structure. Embryo hemodynamics were altered with a 100 nylon suture passed under the outflow tract and tied in a knot around the mid-section of the outflow tract to constrict the crosssectional area. A control group of embryos served as a surgical sham where the suture was passed under the outflow tract but not tightened. Following interventions, eggs were sealed with saran wrap and incubated until further evaluation.

Chick embryos are not considered live vertebrate animals under IACUC and Oregon Health \& Science University regulations until they hatch, however we made every effort to minimize the number of embryos needed.

\section{Band Tightness Measurement with Optical Coherence Tomography (OCT)}

A custom-made optical coherence tomography (OCT) system was used to measure chick embryo band tightness in vivo as previously described (Rugonyi et al., 2008; Ma et al., 2010; Liu et al., 2012; Shi et al., 2013). Briefly, the system has a spectral domain configuration with a superluminescent diode centered at 1,325 $\mathrm{nm}$ from Thorlabs Inc. (Newton, NJ, USA) and a 1,024 pixel, $92 \mathrm{kHz}$ maximal line-scan rate infrared InGaAs line-scan camera from Goodrich Inc. (Charlotte, NC, USA). It acquired $512 \times 512$ pixel, $2 \mathrm{D}$ B-mode tomographic images at 140 frames 
per second with $<10 \mu \mathrm{m}$ resolution. Embryo temperature during OCT acquisition was maintained at a normal physiological temperature $\left(38^{\circ} \mathrm{C}\right)$ with a thermocouple-controlled heating system. Each banded embryo was imaged immediately before and $2 \mathrm{~h}$ after manipulation with OCT to acquire 200 tomographic frames ( 3-4 cardiac cycles) of a longitudinal section of the outflow tract in order to measure the change in outflow tract diameter and calculate the degree of band tightness

$$
\text { Band tightness }=1-D_{a} / D_{b},
$$

where $D_{a}$ is the maximum external diameter of the outflow tract at the band site after banding, and $D_{b}$ is the maximum external diameter of the outflow tract at the approximate band site location before banding. The measured band tightness of each banded embryo was used to define the hemodynamic environment, based on our previous characterization of the relationship between the degree of outflow tract band tightness and the specific blood pressure and velocity conditions induced (Shi et al., 2013; Midgett et al., 2014). Band tightness in this study was limited to $30-45 \%$ constriction, which corresponds to a relatively constant and large (about 3-fold) increase in wall shear rate/stress (Midgett et al., 2014) and moderate (about 1.5-fold) increase in blood pressure (Shi et al., 2013). After OCT imaging, the eggs were re-sealed with saran wrap and placed back in the incubator.

\section{FIB-SEM Acquisition}

Embryos with band tightness between 30 and $45 \%$ constriction were collected at $\mathrm{HH} 24(\sim 24 \mathrm{~h}$ after surgical manipulation at HH18), still corresponding to looping tubular stages of heart development. Samples were processed for focused ion beam scanning electron microscopy (FIB-SEM). FIB-SEM imaging was performed on an FEI Helios 660 NanoLab $^{\text {TM }}$ DualBeam $^{\mathrm{TM}}$. Whole embryos were removed from the egg and immediately immersed in fixative $(2.5 \%$ paraformaldehyde + $2.5 \%$ glutaraldehyde) to maximally preserve and contract the tissue (Damon et al., 2009; Rennie et al., 2014). A portion of the tubular outflow tract containing the endocardial cushions upstream of the banding location was then dissected from the embryo. The fixed tissue was stained and processed as previously described (Rennie et al., 2014), and oriented longitudinally in the resin block face to contain a cross-section of the outflow tract and easily locate the region of interest for imaging. The samples were then processed for FIB-SEM imaging of the myocardium to acquire serial (3D), high-resolution images, as previously described (Rennie et al., 2014). The FIB-SEM system alternated between imaging the block face with a scanning electron beam and milling away $4 \mathrm{~nm}$ thick sections of the block face, in order to collect a high-resolution 3D image volume. A maximum of 1000 slices through the myocardium were acquired on a region of interest with a horizontal field width of $20 \mu \mathrm{m}$.

\section{Image Analysis and 3D Organelle Quantification}

Amira software (FEI) was used for all FIB-SEM image registration, processing and quantification. First, the acquired image stacks were automatically aligned to reconstruct the 3D image volume. Myofibril bundles, mitochondria, nuclear, and extracellular material were then manually segmented (for consistency) from 500 image stacks and reconstructed, in order to quantify organelle 3D volume and organization.

Myofibril and mitochondrial volume fractions in the cell cytoplasm (instead of total cell volume) were used to quantify organelle volume, which accounted for differences in sample nuclear volume that depended on the specific region of interest. Cytoplasm volume was calculated by subtracting the volume of extracellular and nuclear material from the total sample volume. Myofibrils were additionally quantified by bundle thickness and orientation within the outflow tract. Myofibril bundle thickness was used as a measure of the extent of myofibril development, and quantified as the average of measured lengths of Z-lines of all bundles in each sample. Myofibril bundle orientation was quantified in terms of angles $\phi$ and $\theta$ (see Figure 1), where $\phi$ is the angle of the fibril bundle in the xy plane (circumferential plane of the outflow tract, with $\phi=90^{\circ}$ indicating longitudinal alignment and $\phi=0^{\circ}$ indicating circumferential alignment), and $\theta$ is the angle of the fibril bundle in the xz plane (radial plane of the outflow tract, with $\theta=0^{\circ}$ indicating radial alignment and $\theta=90^{\circ}$ indicating circumferential or longitudinal alignment). Both orientation angles were measured for all myofibril bundles defined between Z-lines for each sample. Angle values were scaled so each measure ranged from 0 to $90^{\circ}$, to capture the fact that myofibrils with $5^{\circ}$ and $175^{\circ}$ angle orientation values in a single plane are similarly oriented with respect to longitudinal, circumferential or radial directions. Since the exact orientation of the outflow tract tissue within the resin block for FIB-SEM imaging cannot be controlled, absolute myofibril orientation angle values could not be compared. Instead, the distribution of the orientation angles within each sample was used to analyze the overall organization pattern.

\section{Mass Spectrometry}

In separate experiments, after surgical manipulations at HH18, outflow tracts were dissected from the embryo at $\mathrm{HH} 24$ and rinsed in ultrapure water. There were a total of 5 banded embryo samples (30-45\% band tightness) and 5 sham control samples included in these experiments, with each sample consisting of 8 pooled outflow tracts (40 hearts per treatment distributed into 5 samples). Pooling outflow tracts was necessary to reach the minimum mass required for the mass-spectrometry analysis. Samples were then processed as described before (Midgett et al., 2017a). Briefly, samples were first lysed and sonicated, and lysate protein content determined using a BCA assay (Thermo Scientific) and BSA standard. After further sample processing (involving sample reduction, alkylation, trypsinization, incubation and centrifugation), tandem mass tagging (TMT) reagent (Thermo Scientific) was added to each sample and incubated at room temperature for $1 \mathrm{~h}$. A short single liquid chromatography-mass spectrometry (LC-MS) analysis was then performed to check for complete labeling and to determine the relative summed reporter ion intensities of each sample. This normalization allowed confirmation of $>95 \%$ TMT labeling and generated preliminary summed reporter 

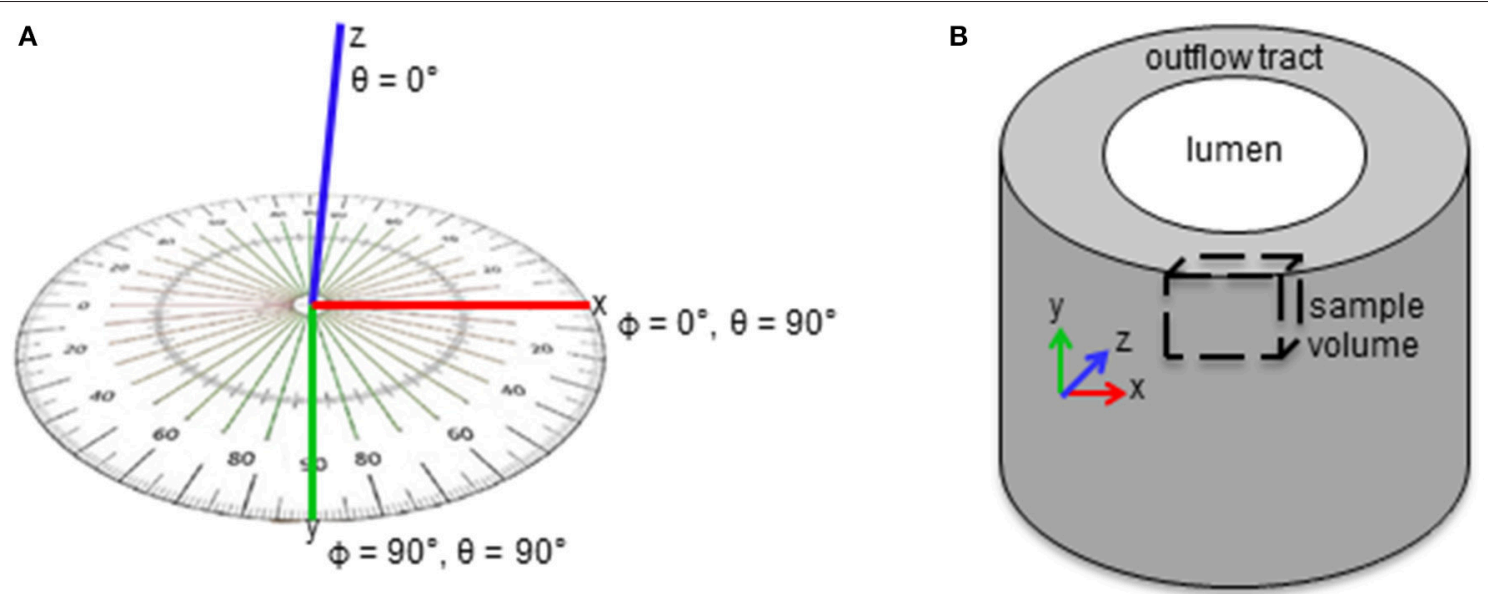

FIGURE 1 | Schematics of myofibril bundle orientation quantification. (A) Angles $\phi$ and $\theta$ were measured for each bundle. (A) $\phi$ and $\theta$ are the angles of the fibril bundle in the xy and xz planes in the sample volume, respectively. (B) $\phi$ and $\theta$ describe the bundle orientation in the circumferential and radial planes of the outflow tract, respectively.

ion intensities for each TMT channel. The 10 samples were then loaded in each channel after adjusting sample volumes so that each sample would yield approximately equal summed reporter ion intensities in the final mass-spectrometry analysis. Tandem mass spectrometry data was collected using an Orbitrap Fusion Tribrid instrument configured with an EasySpray NanoSource (Thermo Scientific). Survey scans were performed in the Orbitrap mass analyzer, and data-dependent MS2 scans in the linear ion trap using collision-induced dissociation following isolation with the instrument's quadrupole. Reporter ion detection was performed in the Orbitrap mass analyzer using MS3 scans following synchronous precursor isolation of the top 10 most intense ions in the linear ion trap, and higher-energy collisional dissociation in the ion-routing multipole.

\section{TMT Data Analysis}

Raw instrument files were processed using Proteome Discoverer version 1.4.1.14 (Thermo Scientific) with SEQUEST HT software and a Gallus gallus Ensembl database (release 85) containing 16,187 sequences. Searches were configured with static modifications for the TMT reagents $(+229.163$ peptide $\mathrm{N}$-terminus and $\mathrm{K}$ residues) and iodoacetamide $(+57.021 \mathrm{C}$ residues), variable oxidation (+15.995 $\mathrm{M}$ residues), parent ion tolerance of $+/-1.25 \mathrm{Da}$, fragment ion tolerance of 1.0 Da, monoisotopic masses, and trypsin cleavage (max 2 missed cleavages). Searches used a reversed sequence decoy strategy to control peptide false discovery and identifications were validated using Percolator software. Only peptides having mass errors $<20 \mathrm{ppm}$, and matching only one protein entry in the database were used. Search results and TMT reporter ion intensities were exported as text files and processed using in-house scripts. After excluding the highest and lowest reporter ion intensities, an average intensity $>500$ was required for the remaining reporter ions to exclude the analysis of peptides with insufficient signal intensity. Reporter ion intensities for peptides assigned to each protein were then summed to create an abundance measurement for each protein across the 10 samples simultaneously analyzed.

\section{Statistical Analysis}

FIB-SEM samples and TMT samples were selected with a relatively small range of band tightness (30-45\%), and therefore differences between samples with varying band tightness were not analyzed. Rather, analysis was performed to determine differences between control and banded samples. Statistical significance between banded and control groups using FIB-SEM image analysis was determined with a two-sample Student's $t$-test, assuming significance with two-tail $p<0.05$ unless otherwise noted.

Differential protein abundance was determined in the TMT experiment by comparing the summed peptide reporter ion intensities for each protein across samples (5 control and 5 banded) using the $\mathrm{R}$ software ( $\mathrm{v}$ 3.1.1) package edgeR (Robinson et al., 2010), which performed data normalization, fold changes, determination of individual protein $p$-values, Benjamini-Hochberg multiple test correction to calculate false discovery rates (FDR or $q$-value) during tests for differential abundance for each protein compared between banded and control samples. These data was used in comparing changes in abundance of specific proteins.

\section{Pathway Analysis}

A pathway analysis of proteomics data was performed using Ingenuity Pathway Analysis (IPA) software (Qiagen Bioinformatics). Protein intensities quantified for each sample (5 control and 5 banded) were used to compute differential protein abundances and protein fold ratios (and their logarithms) between banded and control treatments, as well as $p$-values, and false discovery rates ( $q$-values), as described above using edgeR. We then filtered the data for low intensity values and peptide counts per protein: we discarded protein measurements with low intensity (with at least two of the samples having a 
low intensity reading $<4,000$ ) and proteins obtained using only one peptide. That is, for analysis we kept only proteins that were relatively abundant and measured with accuracy. This rendered 3838 proteins of which 2,544 could be mapped to known proteins/genes in IPA. The filtered data (protein log fold ratios, and $p$-values) were used as input for the IPA analysis (provided as Supplementary file "Data Sheet 1," together with the IPA dataset "Data Sheet 2"). We performed the analysis using the Ingenuity Knowledge Base, a pathway database in IPA, including direct and indirect relationships, considering genes only, endogenous chemicals in network analysis, and with experimentally observed confidence. The analysis was further performed using all node types, available data sources, and all species pathways available, but no mutations considered. Analysis included data from all tissues and cell lines, and was restricted to proteins featuring significant differences in banded and control samples ( $p<0.1$, total 948 proteins analyzed). IPA rendered a list of affected canonical pathways with their $p$-values, which measure statistically significant overlap between the dysregulated input-gene dataset and genes in the pathway, and was calculated using Fisher's exact test. Regulation direction, however, is not considered into the calculation of the pathway $p$-value, and is included instead in the z-scores. The z-score in IPA is defined as

$$
\mathrm{z}-\text { score }=\left(\mathrm{N}_{+}-\mathrm{N}_{-}\right) / \mathrm{N}^{1 / 2},
$$

where $\mathrm{N}$ is the number of detected dysregulated proteins in the pathway, $\mathrm{N}_{+}$and $\mathrm{N}_{-}$are proteins that have changed abundance in the direction of pathway activation and inhibition (according to literature-derived regulation direction incorporated in IPA), respectively. Pathway z-scores therefore quantify activation or inhibition of pathways based on the differential abundance of detected proteins in the pathway.

\section{RESULTS}

\section{Myofibril and Mitochondrial Maturation Is Active at $\mathrm{HH} 24$}

FIB-SEM processing acquired 3D image data from the myocardium layer of the heart at $\mathrm{HH} 24$ with excellent myofibril and mitochondrial ultrastructural detail. Control outflow tract myocytes contained immature myofibril structures (Figure 2), indicating that the development of the outflow tract heart muscle is still ongoing at this stage, as expected. Immature myofibril organization was characterized by the overall lack of myofibril alignment (see Figures 2A,B) and instances where more than one fibril radiated from the same $z$-band center (see Figure 2D). In addition, while most myofibrils inserted into intercalated disks at nearly right angles as they do in mature tissue, some oblique insertions were still visible in control tissue (see Figure 2F). Mitochondria were scattered throughout the cytoplasm with clear walls, cristae, and ribosome structures at this stage of development (Figure 3). Control tissue contained an array of mitochondrial shapes ranging from very long and tubular-like to almost spherical, indicating that mitochondrial maturation is in an active fusion/fission maturation phase. Mitochondria appeared in small groups that were distributed throughout the cytoplasm and around myofibril bundles.

\section{Myofibril and Mitochondrial Volume Fraction Is Unchanged after Increased Hemodynamic Load}

To quantify alterations in myofibril and mitochondrial volume after outflow tract banding, these organelles were segmented from high-resolution FIB-SEM image volumes of myocardial outflow tract tissue. Myofibril and mitochondrial volume fraction in the cell cytoplasm were calculated from the $3 \mathrm{D}$ reconstructions of the segmented images (Figure 4). The myofibril-cytoplasm volume fraction did not significantly vary between control and banded samples ( $12.9 \pm 0.3$ vs. $9.6 \pm 2.8 \%$, in control and banded, respectively; $p=0.17, n=3$ ). Similarly, mitochondrial-cytoplasm volume fraction was comparable in both groups $(10.5 \pm 1.8$ and $9.7 \pm 1.4 \%$ in control and banded; $p=0.56, n=3$ ).

\section{Increased Hemodynamic Load Triggers Myofibril Reorganization and Mitochondrial Misalignment}

3D FIB-SEM reconstructions of myofibril bundle segmentations were used to calculate fibril bundle thickness and orientation. While myofibril volume fraction and orientation in the radial plane ( $\mathrm{x}-\mathrm{z}$ plane in Figure 1 ) did not significantly change after banding, fibril bundles were more clustered; orientation in the circumferential plane ( $\mathrm{x}-\mathrm{y}$ plane in Figure 1$)$ was more distributed, and bundles were thicker following increased hemodynamic load. Three-dimensional reconstructions from the FIB-SEM samples (Figure 4) qualitatively show that the segmented myofibril material was more clustered in banded samples compared to controls where the myofibril material was distributed throughout the cells. Myofibril bundle orientation was quantified in terms of angles $\phi$ and $\theta$ (see Figure 1), where $\phi$ is the angle of the fibril bundle in the $x-y$ plane (circumferential plane of the outflow tract sample), and $\theta$ is the angle of the fibril bundle in the $\mathrm{x}-\mathrm{z}$ plane (radial plane of the outflow tract sample). The $\phi$ myofibril angles in control myocardium concentrated in the region of low $\phi$ with an average of $42.6 \%$ of myofibrils within a sample concentrated within a 20 degree range of $\phi$, indicating more predominant fibril alignment in the circumferential direction $(\phi=0)$. In contrast, the fibril orientation in this plane in banded samples was more distributed and contained fibers that pointed in both circumferential and longitudinal directions of the outflow tract myocardium (Figure 5), with an average of only $24.7 \%$ of myofibrils concentrated within a 20 degree range of $\phi(\phi 0$ $20^{\circ}$ ). The distribution of $\theta$ angles in the myofibrils was similar between control and banded samples and indicated a preferential distribution of the fibers in the circumferential direction of the outflow tract $\left(\theta=90^{\circ}\right)$.

Additionally, the average bundle thickness across samples in each group was statistically larger in banded samples ( $p$ $<<0.01, n=3$ ). Box plots show that there was a larger 


\section{A}

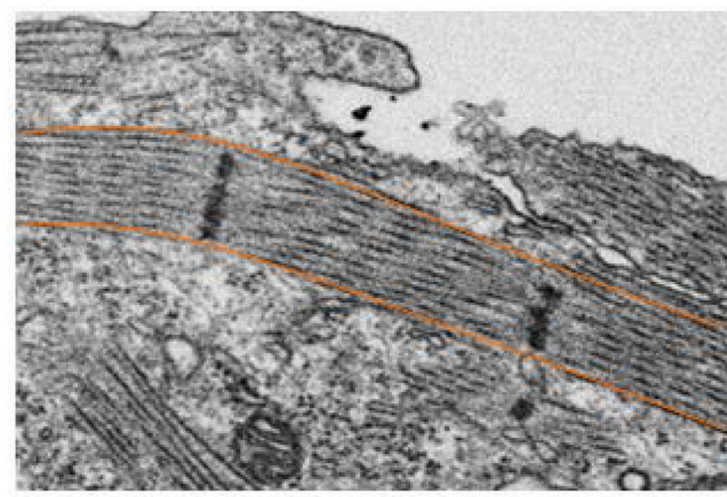

C

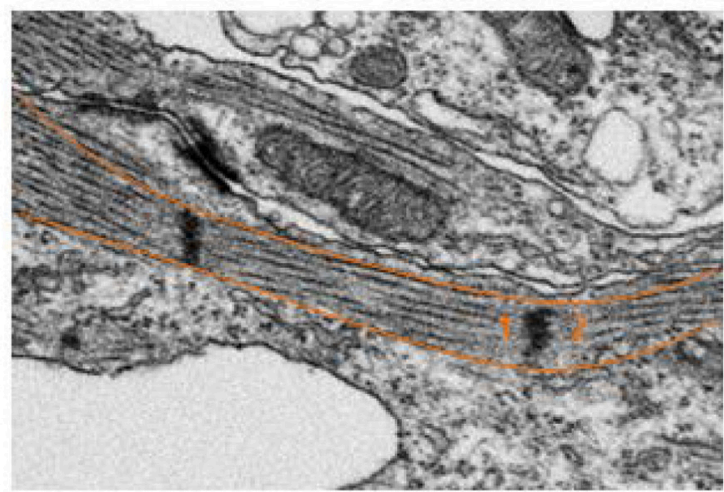

E

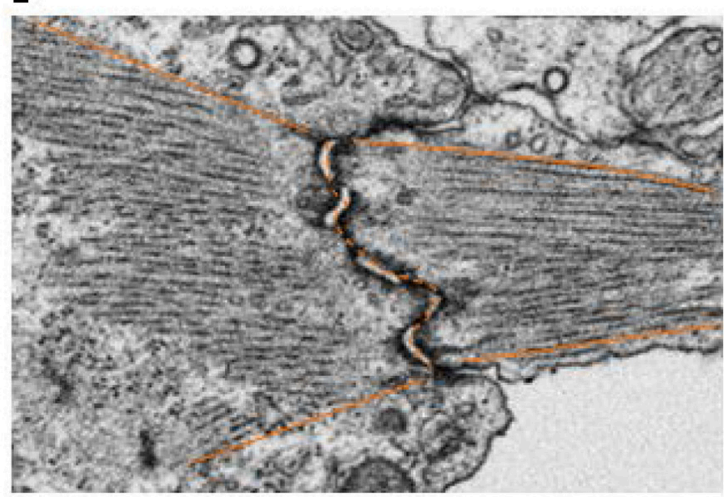

B

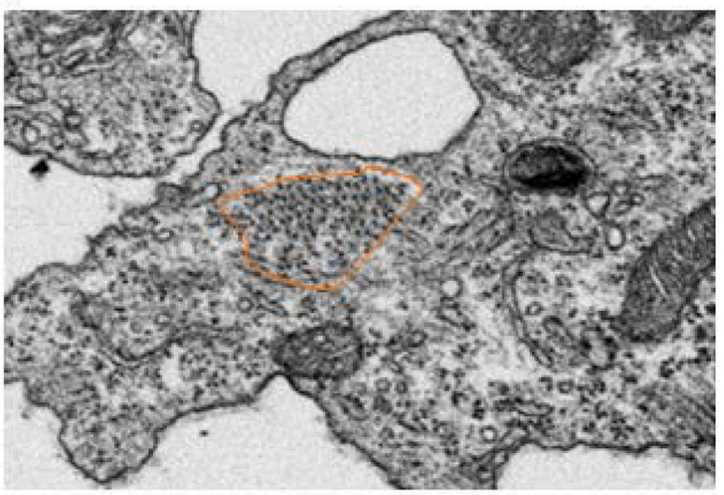

D

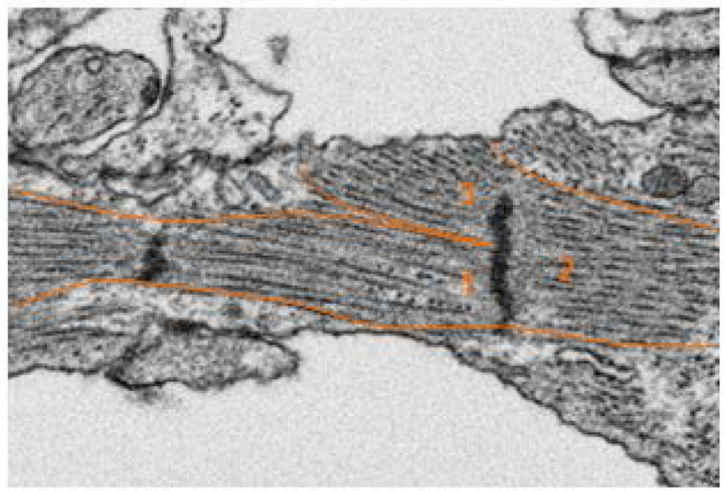

$\mathbf{F}$

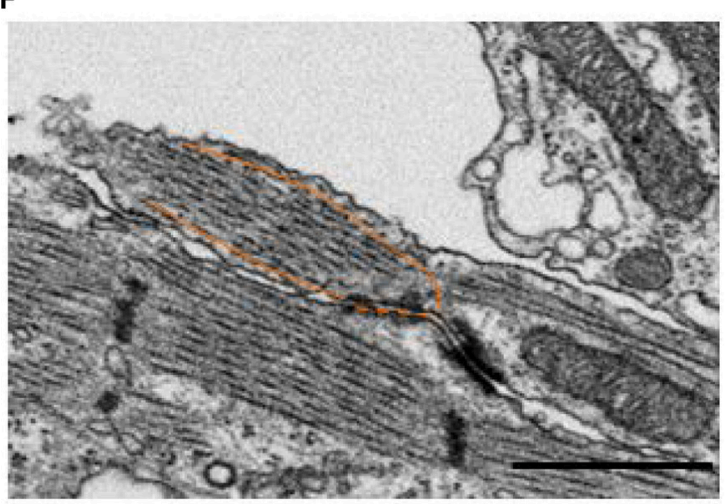

FIGURE 2 | Example FIB-SEM image showing myofibrils in control outflow tract myocardium tissue. Several myofibril orientations are displayed, where the tissue mostly consisted of longitudinally oriented bundles arranged circumferentially around the outflow tract (A) compared to less frequent radially oriented bundles (B). The majority of z-bands only had a single myofibril bundle extending from either side (C), however, samples also contained a few examples of an immature configuration where 3 or more fibril bundles radiated from the same z-band center (D), as numbered above. Most myofibrils inserted into intercalated disks at nearly right angles as they do in mature tissue $\mathbf{( E )}$, while some oblique insertions were still visible in control tissue $\mathbf{( F )}$. Scale bar $=1 \mu \mathrm{m}$.

range of bundle thicknesses in banded samples, indicating that these samples had both small and abnormally large bundles (Figure 6).

Despite unchanged mitochondrial volume fraction in banded samples compared to controls, qualitative differences in the mitochondrial arrangement were displayed in FIB-SEM 3D reconstructions (Figure 4). Control and banded tissue contain an array of mitochondrial shapes ranging from very long and tubular-like to almost spherical, indicating that mitochondrial maturation is active. Mitochondria, however, appeared more clustered in banded samples where each sample showed 2-3 main groups of mitochondria that were largely void of myofibrils (see Figure 4). While there were still clusters of mitochondria in control samples, they clustered in smaller groups and were overall more distributed throughout the myofibrils. 


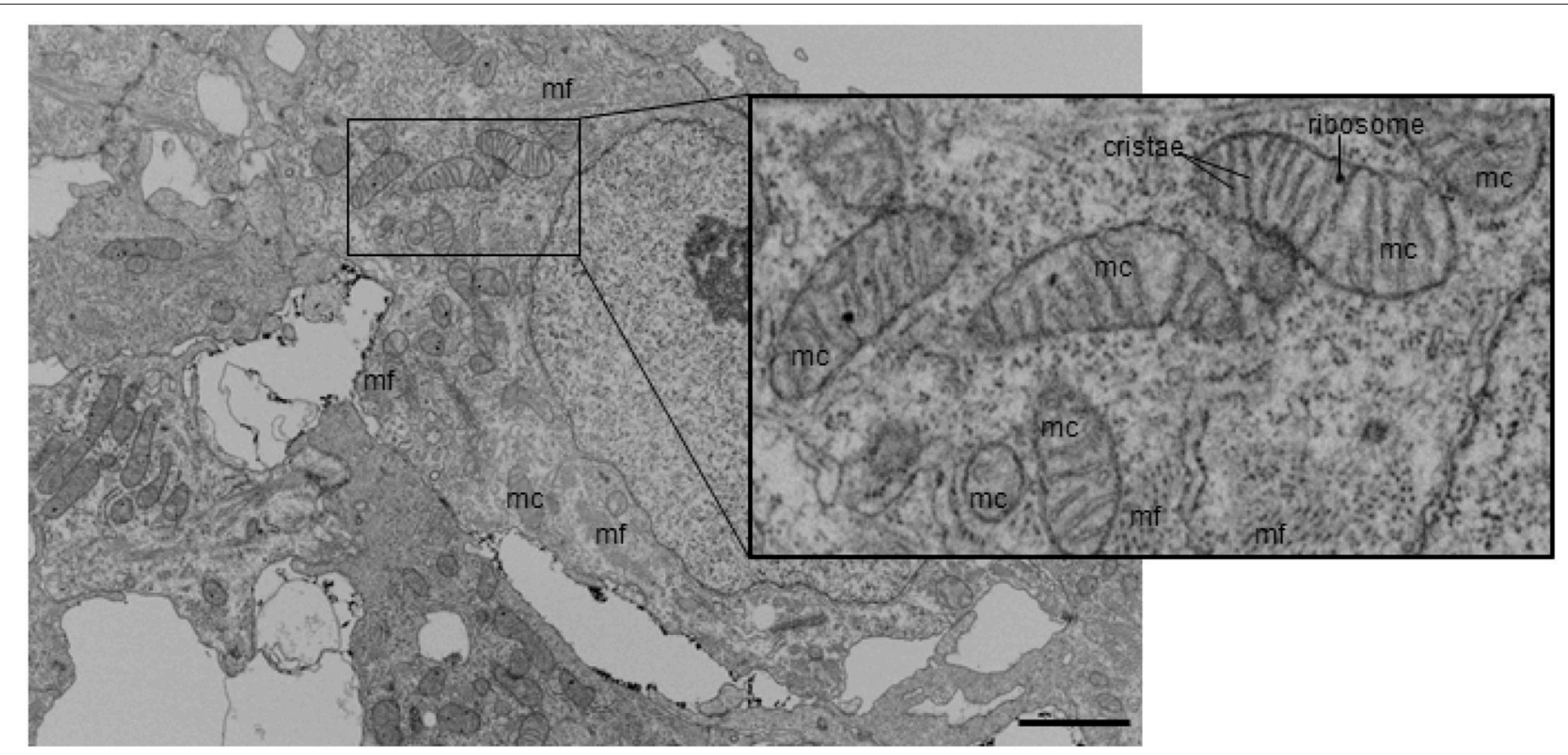

FIGURE 3 | Example FIB-SEM image showing mitochondria among myofibrils in control outflow tract myocardium tissue. Myofibrils are arranged in both circumferential and radial orientations. Mc, mitochondria; $\mathrm{mf}$, myofibril. Scale bar $=5 \mu \mathrm{m}$.
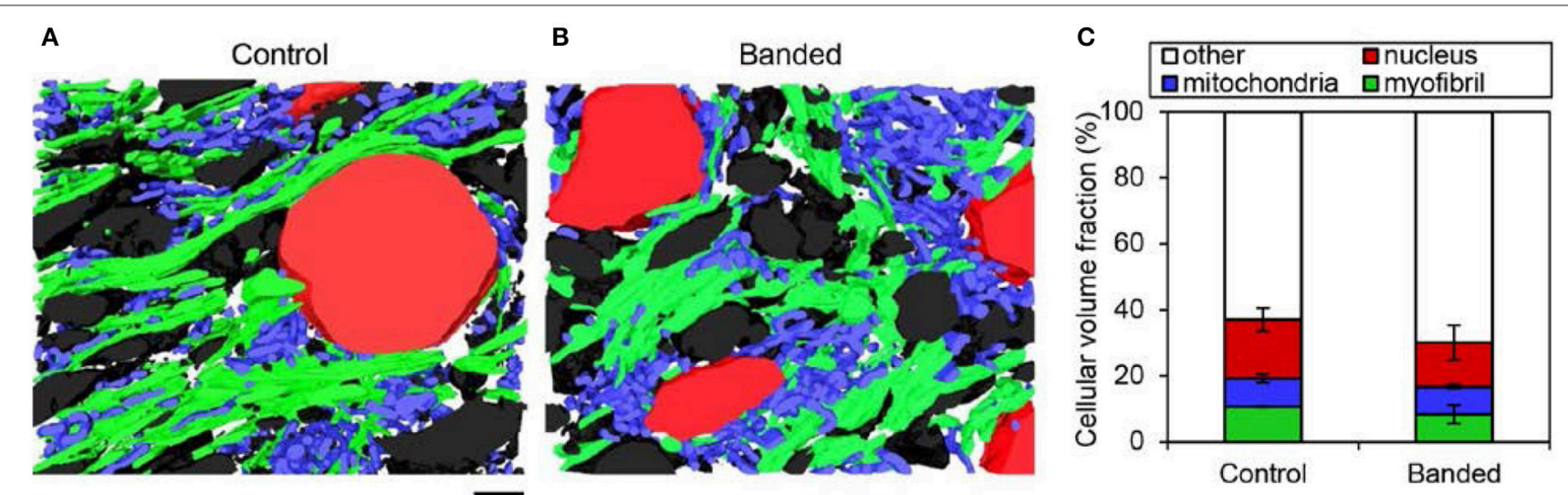

FIGURE 4 | FIB-SEM organelle volume quantitation summary. Example 3D myocardium segmentation reconstructions from a control (A) and banded (B) embryonic heart with myofibril, mitochondrial, nuclear, and extracellular material displayed in green, blue, red, and black, respectively. (C) Average cellular volume fraction quantification from 3D reconstructions ( $n=3$ per group), where there were no significant differences of any cellular component between banded and control samples. Scale bar $=2 \mu \mathrm{m}$.

\section{Increased Hemodynamic Load Triggers Myofibril and Mitochondrial Proteomic Response}

TMT based mass spectrometry provided a proteome-wide assessment of changes in the relative abundance of specific proteins induced by increased hemodynamic load. Specifically, we first focused on examining changes in proteins known to be markers of myofibril and mitochondrial reorganization. A total of 5,330 proteins were detected in the outflow tract samples, where the abundance of 606 proteins significantly differed between banded and control samples (false discovery rate, $q<0.1, n=5)$. The full proteomics data set was deposited in the PRIDE repository database (ProteomeXchange Consortium), with dataset identifier PXD005362. A volcano plot of the data is shown in Figure 7, with point in red listed in the Supplementary file "Data Sheet 3." Of the proteins that were significantly up or downregulated, 3 proteins were related to myocardial contraction, and 17 were directly related to mitochondrial form and function. All proteins had at least 2 peptide-spectrum matches with reporter ion signal used in quantitation among our samples. The main protein changes 

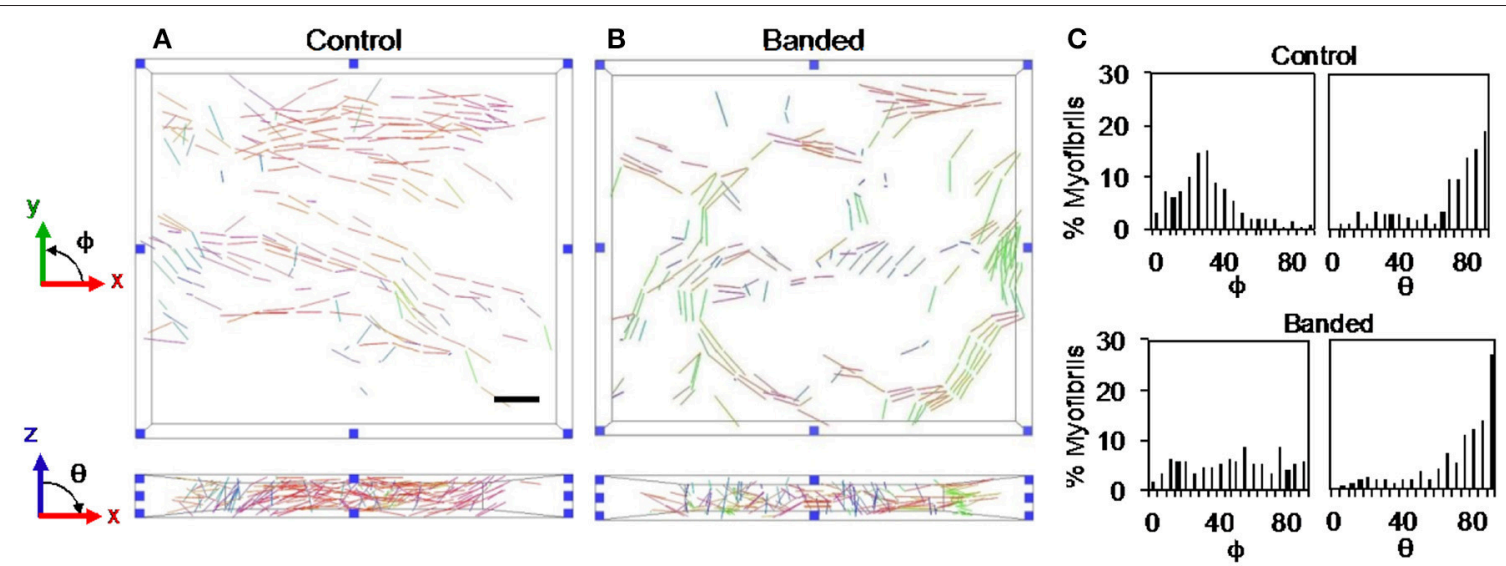

FIGURE 5 | Example myofibril bundle orientation display where each bundle is shown colored based on the orientation in the xy and xz planes for a control (A) and a banded (B) embryo. (C) Example myofibril bundle orientation angle histograms for a control and banded embryo. Scale bar $=2 \mu \mathrm{m}$.
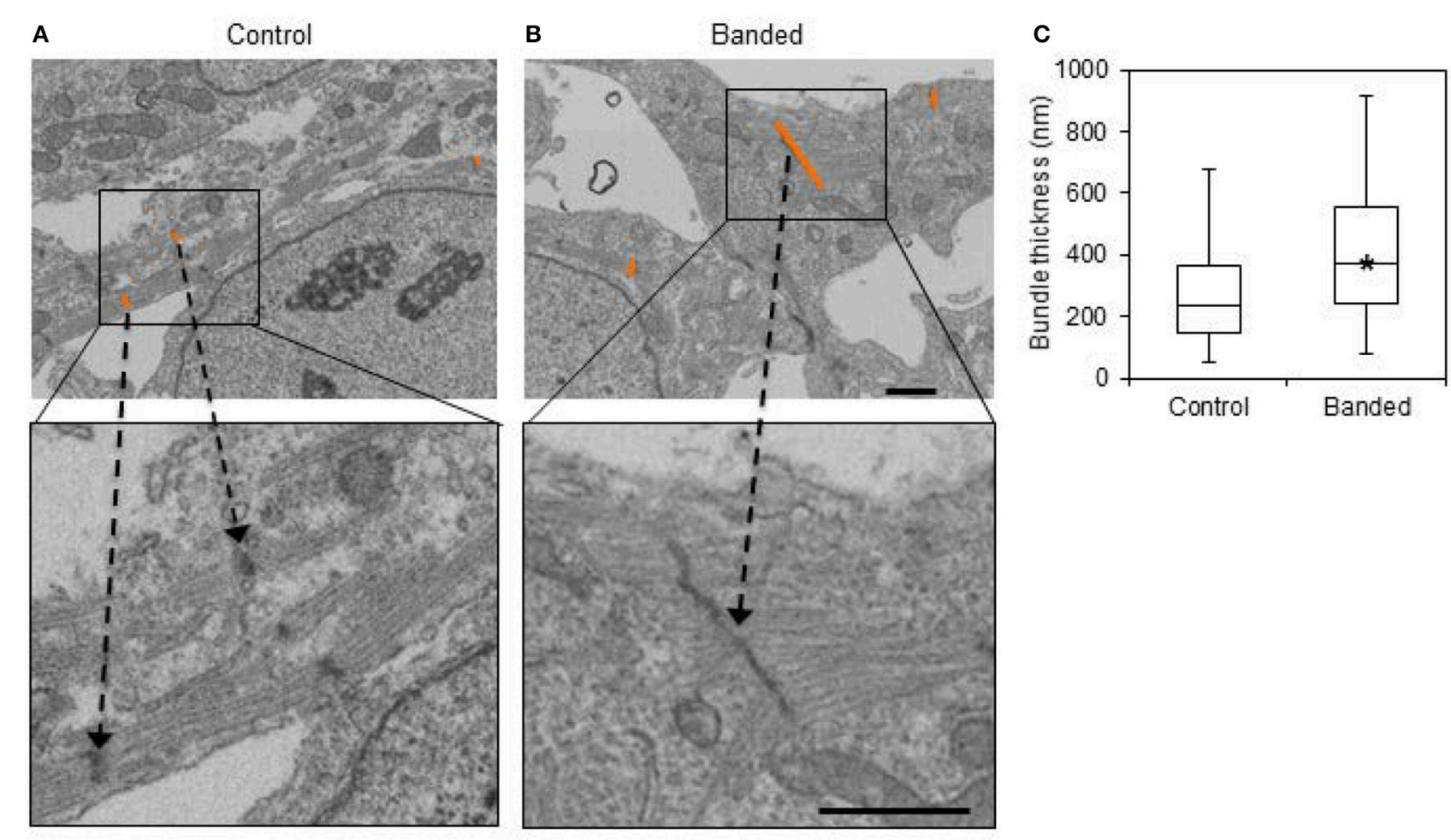

FIGURE 6 | Example FIB-SEM images from a control (A) and a banded (B) embryo, with example bundle thickness measurement lengths at z-bands shown in orange. (C) Bundle thickness quantification box plots displaying the average, with boxes that mark the upper and lower quartiles and whiskers that mark the maximum and minimum values. The asterisk designates a significantly higher bundle thickness average in banded samples compared to controls $(p<0.05, n=3)$. Scale bar $=1 \mu \mathrm{m}$.

included components of myocardial thin filament assembly and function; and mitochondrial maintenance, organization, and the electron transport system (Figure 8). Table 1 lists changes in proteins related to myocardial contractile proteins and Table 2 lists changes in cardiac mitochondrial proteins. Each table gives the protein fold changes between control and banded samples and lists each protein function in terms of myocardial reorganization. Most fold-changes in protein abundances are relatively small (1.1- to 1.5 -fold), which may reflect a modulation of responses by banding and the relatively short intervention time span $(\sim 24 \mathrm{~h})$. Overall proteomics data indicates an increase in proteins associated with myocardial contraction and 


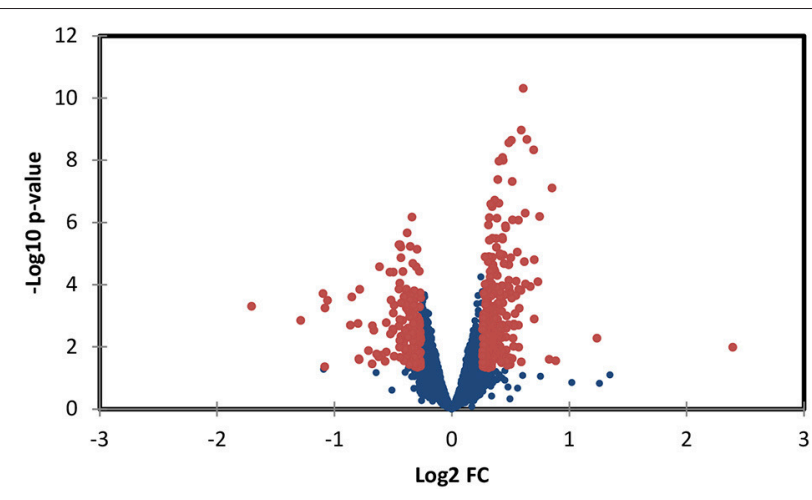

FIGURE 7 | Volcano plot of proteomics data, depicting protein data $p$-values vs. fold change (FC). Red points represent data points with $p<0.05$ and a fold rate $>1.2$; proteins associated with these points are listed in the supplementary file "Data Sheet 3."

mitochondrial clustering; and a decrease in proteins associated with mitochondrial fusion, electron transport, and metabolic function.

\section{Increased Hemodynamic Load Affects Pathways Related to Mitochondrial Function and Stress Response}

A pathway analysis of proteomics data using IPA software resulted in a list of canonical pathways differentially affected by banding. We first selected pathways that were significantly affected (pathway $p<0.05$ ), and that were either significantly activated or inhibited ( $\mathrm{z}$-score absolute value $>2.0$ ), when performing the analysis using only dysregulated proteins with $p$ $<0.1$ (see Figure 9). A detailed graphical depiction of pathways of interest is shown in the Supplementary file "Presentation 1;" while lists of dysregulated genes per pathway are shown in the Supplementary file "Data Sheet 4." Note, however, that in general the ratio of detected dysregulated molecules per pathway with respect to pathway molecules is small, likely due to limitations in the proteomics approach that did not detect less abundant proteins as well as our filtering prior to analysis. The inhibition of the "Remodeling of Epithelial Adherens Junctions" pathway is likely related to the changes in endothelial mesenchymal transition (EMT) that we have reported before (Midgett et al., 2017a), where there is an increased cell density in outflow tract cardiac cushions with banding vs. control, presumably due to increased EMT.

Except for the RAS pathway, other pathways selected exhibited changes in abundance in downstream proteins, but not in the proteins and receptors initiating the signaling cascades. While these pathways regulate cell behaviors (e.g., cell migration and proliferation) and could indeed be indispensable for cardiac development (e.g., Sonic Hedgehog Signaling), validation would be required to show pathway activation/inhibition in response to banding.

In the RAS pathway, we found increase in abundance of upstream regulators. In particular, we found an increase in angiotensinogen (AGT; fold change 1.3, $p<2 \cdot 10^{-4}$ ) and the angiotensin I converting enzyme (ACE; fold change 1.2, $p<0.02$ ). These upstream effectors initiate signaling through angiotensin II (Ang II) after converting AGT into Ang I and then Ang II. Downstream proteins, in addition, were also affected. In particular STAT3 increased 1.2-fold in banded hearts compared to controls $(p<0.002)$. Nevertheless, due to the relatively low number of proteins detected in the RAS pathway, validation of pathway activation needs to be performed in future research. RAS is known to play an important role in the response of the fetal and mature heart to pressure, and its involvement could be pivotal in heart development.

Other canonical pathways highlighted in our analysis were the "Mitochondrial Dysfunction," "EIF2 Signaling," and "Oxidative Phosphorylation" pathways (all with $p<<0.001$ ). Both the Mitochondrial dysfunction and Oxidative phosphorylation pathways are related to regulation of mitochondrial function and showed a reduction in components of the mitochondrial complexes I, IV, and V, as well as a (a weaker) reduction in Complex III. While z-scores for these pathways were not available since there was not enough information on the IPA database to determine pathway activation status, the Molecule Activity Predictor (MAP) tool available in IPA predicted decrease of ATP production associated with these pathways. The EIF2 signaling pathway, in contrast, was activated (z-score 1.9) and it is associated with global protein synthesis and regulation of mRNA. MAP applied to the EIF2 signaling pathway predicted increased cardiac protection, as well as increase amino acid transport and synthesis. Interestingly, and in connection with mitochondrial function, we found significant decreases in the cAMP-responsive element binding 1 (CREB1) protein (fold change $0.8, p<0.003$ ); and in the mitochondrial transcription factor A (TFAM; fold change $0.9, p<0.02$ ).

\section{DISCUSSION}

Myofibril and mitochondrial organization in the myocardium of the early embryonic outflow tract was analyzed after increasing hemodynamic load via a surgical intervention. Outflow tract banding increases blood pressure throughout the circulatory system (Tobita et al., 2002; Shi et al., 2013) and blood flow velocities in the outflow tract (Rugonyi et al., 2008; Midgett et al., 2014). Increase in blood pressure and flow velocity (and the associated increase in wall shear rates/stresses) are dependent on the degree of band tightness (Shi et al., 2013; Midgett et al., 2014). Our previous investigations showed that peak (maximum) blood flow velocity increased with band constriction up to $~ 35 \%$ band tightness, above which it plateaued and decreased, while peak ventricular pressure increased approximately exponentially with constriction. The band constriction range of 30-45\% used for this study corresponds to maximal increase in flow velocity induced by banding ( $\sim 3$-fold increase in wall shear rates), while blood pressure increase was more moderate $(\sim 1.5$-fold from control). Furthermore, these blood flow conditions described by the degree of band constriction lead to specific abnormal cardiac structure and function in later stages of development, including ventricular septal defects and double outlet right ventricle defects (Midgett et al., 2017b). This study describes the earliest remodeling 


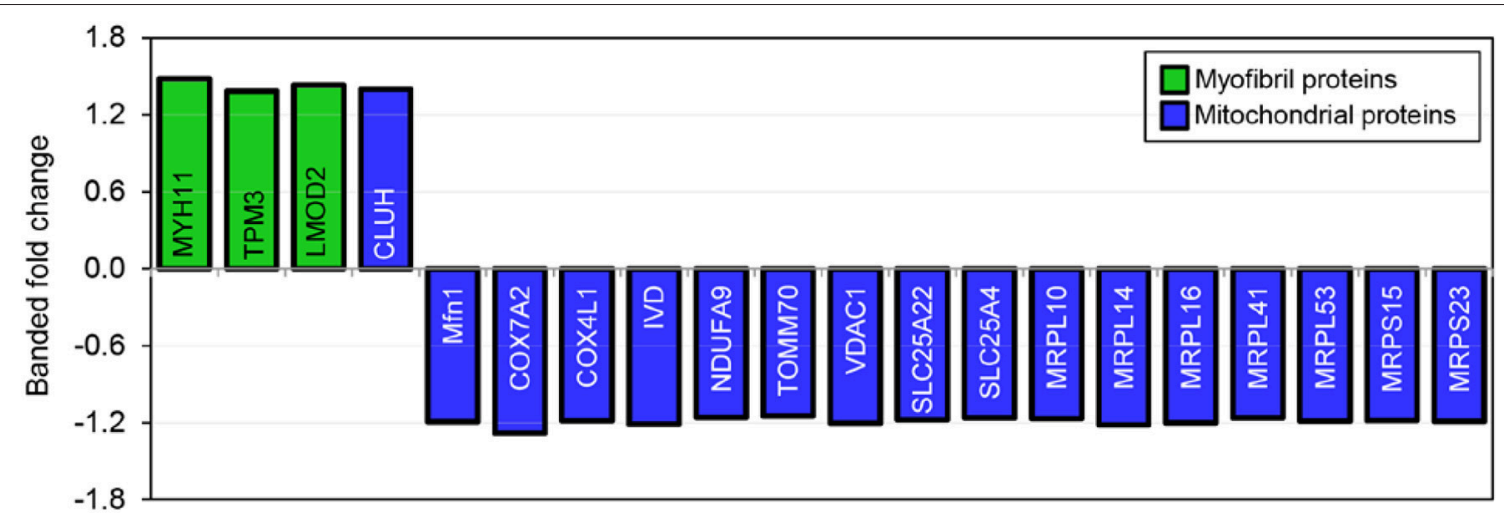

FIGURE 8 | Fold changes in mass-spectrometry measured protein abundances in banded samples compared to controls. Proteins associated with myofibril and mitochondrial proteins are shown, with positive and negative fold changes representing protein upregulation and downregulation, respectively. Protein abbreviations are listed in Tables 1, 2.

TABLE 1 | Myocardial contractile proteins.

\begin{tabular}{|c|c|c|c|}
\hline & Banded fold change & $q$-value & Function \\
\hline Myosin, heavy chain 11 , smooth muscle (MYH11) & +1.5 & 0.04 & Contractile protein of smooth muscle cells (England and Loughna, 2013). \\
\hline Tropomyosin 3 (TPM3) & +1.4 & 0.03 & $\begin{array}{l}\text { Member of the tropomyosin family of actin-binding proteins that are central } \\
\text { to the control of calcium-regulated thin filament function and striated muscle } \\
\text { contraction (Rajan et al., 2010; Bai et al., 2013). }\end{array}$ \\
\hline Leiomodin 2 (LMOD2) & +1.4 & $<0.01$ & $\begin{array}{l}\text { Actin-binding protein that promotes the regulation of striated muscle thin } \\
\text { filament assembly (Tsukada et al., 2010; Pappas et al., 2015). }\end{array}$ \\
\hline
\end{tabular}

q-value: false discovery rate adjusted $p$-value.

effects involved in the initiation of harmful tissue remodeling that eventually lead to cardiac defects. While several authors studied the response of the myocardium ventricle to load, in this work we chose the heart outflow tract since ultimately most cardiac defects in humans have their origins in this cardiac segment.

Myofibril and mitochondria were segmented from FIBSEM image volumes, in order to reconstruct $3 \mathrm{D}$ organelle structures and analyze the architecture of mitochondrialmyofibril networks. Changes in organelle volume fraction and organization were evaluated in outflow tract banded and control myocardial tissue samples to elucidate the effects of increased hemodynamic load on myofibril and mitochondria. While altered blood flow likely triggers spatially and temporally dependent effects along the length of the tubular heart over the course of development, the FIB-SEM samples in this study were from a single location upstream of the banding site near the outflow tract cushions at a single embryonic stage $\sim 24 \mathrm{~h}$ after the hemodynamic intervention. Therefore, our study highlights early changes in the outflow tract tissues in response to banding that likely affects further heart development and malformation at later stages.

Even though myofibril and mitochondrial volume fraction is unchanged after increased hemodynamic load, altered myofibril and mitochondrial organization suggest that abnormal hemodynamics induced by banding interferes with the normal myocardial cell maturation process. Unchanged organelle volume fractions after banding is consistent with a study by Clark et al. that found similar organelle proportion of myofibrils and mitochondria, DNA-to-protein ratio, and myocyte area after outflow tract banding at $\mathrm{HH} 21$ and concluded that measured increases in ventricular weights were due to myocyte hyperplasia (Clark et al., 1989). Nevertheless, banded samples had both thicker myofibril bundles that were more randomly aligned in the myocardium, and mitochondria that were organized in larger clusters compared to controls. While the number of FIB-SEM samples in this study was small because of the limitations of the extensive imaging and analysis required $(n=3)$, these results taken together with the proteomic data indicate an organizational myofibril and mitochondrial response to increased hemodynamic load.

Although, previous studies have characterized myofibril morphology during development, the mechanisms of myofibril formation and alignment are still poorly understood (Sanger et al., 2005). It has been postulated, however, that fibrils align in specific regions of the heart in response to mechanical contraction stresses that increase throughout development in chicken embryos (Manasek, 1970). Raeker et al. suggested that fibril bundles slide to align z-disks as well as dismantle and reassemble to aid in bundling small myofibril clusters to form larger bundle units in skeletal muscle in zebrafish. Further, they concluded that degradation in myofibril organization and reorganization of bundles during development seemed to occur when the offset between z-disks was too large to permit intact integration (Raeker et al., 2014). Differences in $\phi$ orientation 
TABLE 2 | Cardiac mitochondrial proteins.

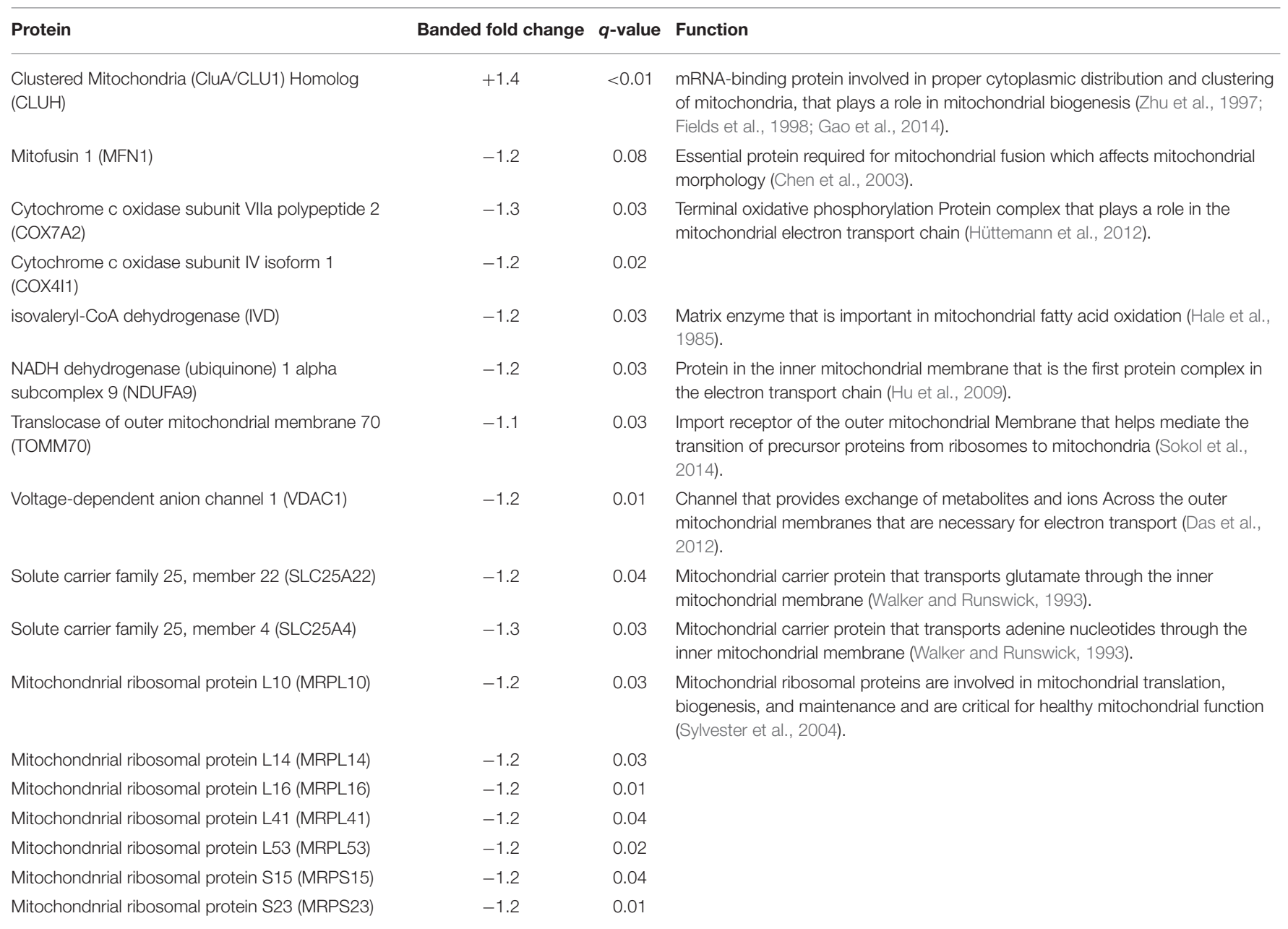

$q$-value, false discovery rate adjusted $p$-value.

angle distributions in our samples may be due to similar shifting of myofibrils to cluster and align, which results in thicker bundles. These changes in myofibril organization and size suggest that increased hemodynamic load induced by banding may accelerate the normal myocardial maturation process.

FIB-SEM reconstructions from control samples showed mitochondria that appear in small groups that were distributed throughout the cytoplasm and around myofibrils, while mitochondria in banded samples mainly clustered in $\sim 2-3$ large groups per sample that were largely void of myofibrils. The large mitochondrial clusters in banded samples were arranged around the nuclei, which is consistent with a progressed stage of mitochondrial organization in embryonic development. Mitochondria are normally scattered throughout the myocyte in early embryonic stages, but as the myofibrils become more dense, mitochondria aggregate around the poles of the nucleus and between myofibrils, and ultimately arrange in a lattice organization throughout the myofibrils (Brook et al., 1983; Vendelin et al., 2005; Chung et al., 2007). Changes in the mitochondrial organization after outflow tract banding indicate that increased hemodynamic load causes mitochondria to conform to a more mature configuration.

Mass-spectrometry was used to detect global changes in protein abundances after banding to reflect the overall dynamic regulation balance between transcription, localization, modification, and programmed destruction of protein during myocardial reorganization in response to altered hemodynamic load. The proteomic analysis corroborates the FIB-SEM evaluations, where we found that increased hemodynamic load triggers a proteomic response corresponding to key myofibril and mitochondrial functional proteins (see Figure 8 and Tables 1, 2). Altered myofibril proteins included components of thin filament assembly and function. Proper contraction of overlapping arrays of actin-containing thin filaments and myosin-containing thick filaments in cardiomyocytes requires appropriate filament alignment. Accordingly, their orientations, spacing, and lengths throughout development are highly regulated by several proteins (Tsukada et al., 2010). For example, Tropomyosin 3 stabilizes filamentous actin and coordinates the actomyosin interaction (Rajan et al., 2010; Bai et al., 2013), while leiomodin 2 is 


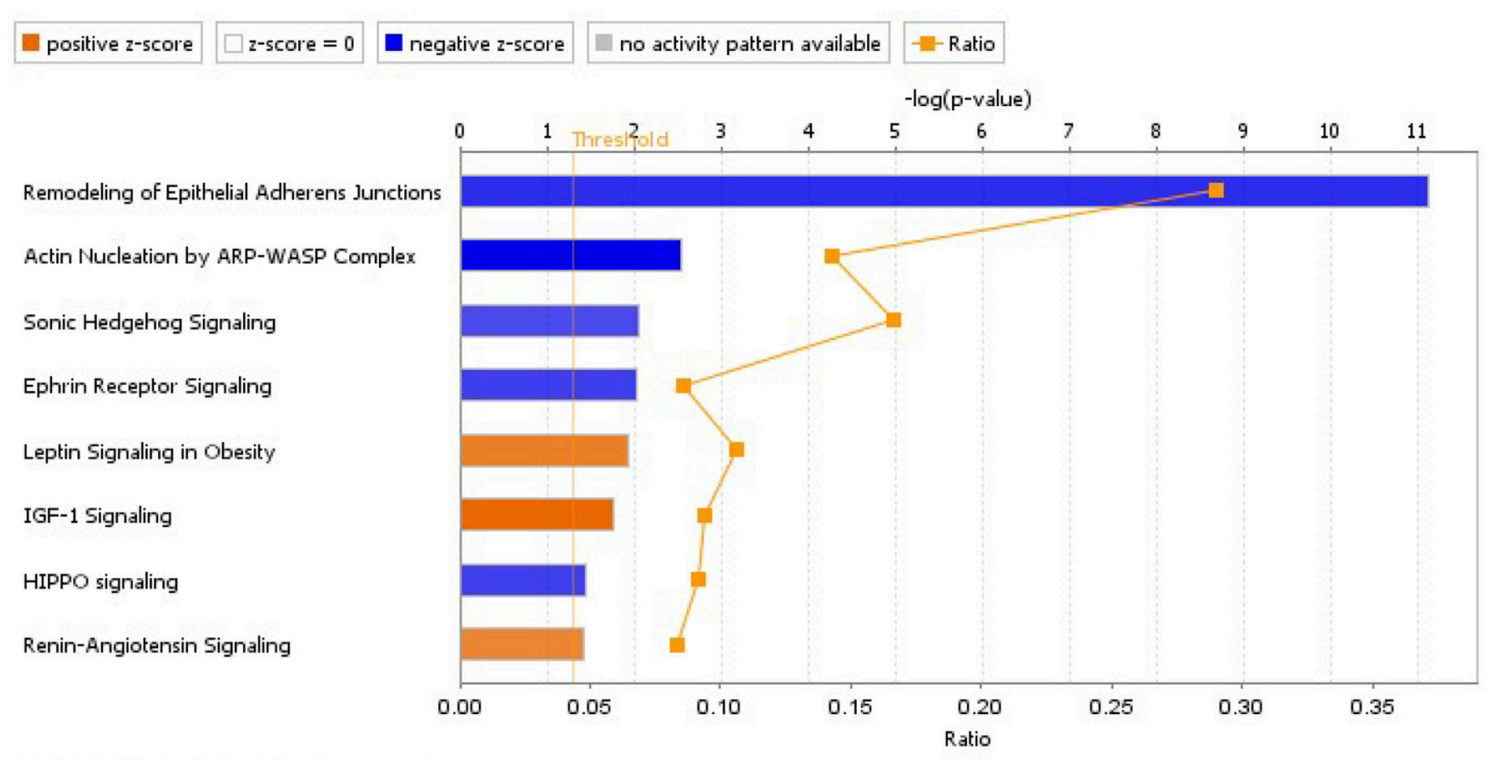

FIGURE 9 | Significantly inhibited and activated pathways due to banding treatment, based on proteomics data. The analysis was performed in IPA, using data from proteins that changed abundance significantly $(\rho<0.1 ; 948$ proteins included in the analysis), and considering information from all tissues/cell lines. Pathways plotted have $p<0.05$ and $z$-score absolute value $>2$ (blue denotes inhibition and orange activation). The plotted ratio shows the ratio of the number of dysregulated molecules in our analysis data set that corresponded to the pathway, vs. the total number of molecules in the canonical pathway.

associated with the promotion of actin assembly and thin filament elongation (Pappas et al., 2015). Myosin heavy chain 11, tropomyosin 3, and leiomodin 2 proteins were upregulated in banded samples compared to controls, indicating that these proteins likely work in concert to help produce the changes in myofibril bundle orientation distributions and increased bundle thickness detected with the 3D FIB-SEM analysis.

Analysis of mass-spectrometry data combined with pathway analysis, revealed increases in the abundance of proteins associated with the RAS pathway with banding. In adults, RAS is a key regulator of blood pressure (Te Riet et al., 2015), with its upregulation in the heart leading to cardiac fibrosis and heart failure (Schmieder et al., 2007; Knight et al., 2012). The role of RAS in early cardiac development, however, has been less studied and still needs to be fully elucidated (Price et al., 1997). Our proteomics analysis revealed upregulation of AGT and ACE after $24 \mathrm{~h}$ of banding. Activation of the RAS pathway starts with conversion of AGT to angiotensin I (Ang I) by renin, and then Ang I is converted to Ang II by ACE. Angiotensin II then activates the angiotensin receptors 1 or 2 (AT1R or AT2R). In the adult heart, Ang II acts predominantly through AT1R inducing myocardial hypertrophy and fibrosis; with increased hemodynamic load leading to increase Ang II levels (Price et al., 1997). In neonatal pigs, Ang II is required for normal cardiac development (Beinlich and Morgan, 1993; Beinlich et al., 1995). In studies using rats, embryos were allowed to develop to embryonic day 9.5 in vivo (tubular heart stages) and then they were cultured for $48 \mathrm{~h}$. Addition of Ang II in the cultured media resulted in increased myofibril development in the heart. Ang II treated hearts exhibited more advanced sarcomere and Z-band organization, which resembled the increased myofibril development that we observed in our banded hearts with respect to controls. Thus our data, in view of previous studies, may imply that myofibril reorganization after increased hemodynamic load in banded hearts may be mediated by RAS. While this relationship will need to be further validated, especially given the low number of proteins in RAS detected by the proteomics analysis, it is certainly an exciting possible direction of research.

We also found increased abundance of STAT3 in banded hearts vs. control hearts. STAT proteins are expressed in the mature heart, and in particular STAT1 and STAT3 play roles in ischemic heart disease, with STAT1 contributing to the decrease of myocardial cells by increasing apoptosis, while STAT3 being cardioprotective by decreasing reactive oxygen species, but related to early remodeling of the heart that then leads to heart failure (Knight et al., 2012). In our embryonic banded heart outflow tracts, STAT3 was increased compared to controls, but STAT1 and STAT5B, while detected in the proteomics analysis, did not change significantly with banding. STATs are recruited and activated by the Janus kinase (JAK) family, and translocate to the nucleus where they transactivate target genes (Knight et al., 2012). Previous studies, have also demonstrated that the JAK-STAT pathway is also involved in the RAS pathway, mediating gene transcription (Satou and Gonzalez-Villalobos, 2012). Further, STAT3 has been shown to play a regulatory role inside mitochondria, regulating mitochondrial electron transport (Szczepanek et al., 2011; Pohjoismäki et al., 2012). In our banded hearts, as blood pressure increases due to introduction of the band, therefore, mechanisms that respond to hemodynamic overload seem to take place, including enhanced activation of RAS and increase in the cardioprotective STAT3. 
Several mitochondrial protein abundances were altered in banded samples including components of mitochondrial maintenance, organization, and the electron transport system. Proteins with roles in mitochondrial fission or fusion and cell death were detected by the TMT experiment, although they were largely unchanged in banded samples compared to controls. These proteins included Dynamin-1-like protein, optic atrophy type 1 , mitochondrial fragmentation-causing deathassociated protein 3, and dynamin-related protein 1 (Chen et al., 2003; Chung et al., 2007). However, mitofusin 1 was slightly downregulated in banded embryos compared to controls by 1.2-fold, indicating that changes in mitochondrial fusion after banding may be partly responsible for the organizational changes observed in the 3D FIB-SEM reconstructions. Differential protein abundance of Clustered Mitochondria Homolog (CLUH) in banded samples was also detected by proteomic analysis. CLUH silencing in mammalian cells and other species alters the mitochondrial cellular distribution and leads to mitochondrial clustering in the center of the cell (Zhu et al., 1997; Fields et al., 1998; Gao et al., 2014). The clustered groups of mitochondria observed in the FIB-SEM reconstructions combined with a significant increase in CLUH protein of banded samples indicates that perhaps CLUH has an alternate role in mitochondrial biogenesis in embryonic development. The proteomic analysis also showed varied abundance of 5 large and 2 small mitochondrial ribosomal proteins (MRPs). While MRPs are not generally well-conserved among species and the roles of specific MRPs in chick cardiac development are largely unknown, the integrity of healthy mitochondrial function is thought to largely depend on normal MRP-dependent protein biosynthesis, translation, and maintenance (Sylvester et al., 2004). Proteomic response in banded samples was also detected for components of mitochondrial energy generation, including electron transport complexes, ion channels, and solute carrier proteins. Proper mitochondrial energy generation is essential for the functional health of cardiac tissue (Hüttemann et al., 2012), which may be disrupted as part of the mitochondrial reorganization in banded tissue. Other than CLUH, however, all other mitochondrial-related proteins were downregulated, suggesting a decrease in mitochondria or in mitochondrial function. Since mitochondrial cytoplasmatic volume is conserved in our studies, this downregulation is likely related to function. In fact, MAP applied to the IPA Mitochondrial Dysfunction canonical pathway predicts decrease of mitochondrial function and ATP. Mitochondria function depends on transcriptional regulators and nuclear-encoded mitochondrial (NEM) proteins. Since the great majority (99\%) of mitochondrial proteins are encoded in the nucleus, transport of cytosolic proteins plays an important role on mitochondrial function (Boengler et al., 2011). Many NEM proteins are regulated by cAMP-responsive element binding (CREB) proteins. Interestingly, in our proteomics dataset we found a significant decrease in CREB1. The mitochondrial transcription factor A (TFAM), in addition, is a major regulator of mitochondrial DNA (mtDNA) copy number and is required for mtDNA transcription, and thus plays an important role on mitochondrial maintenance (Pohjoismäki et al., 2012). In banded heart outflow tracts, however, TFAM abundance decreased. Thus, while myofibrils and mitochondria seem more clustered in banded hearts, this could be at the expense of mitochondrial function.

The EM imaging and proteomic data taken together indicate that hemodynamic forces induced by banding after only $24 \mathrm{~h}$ of intervention interfere with normal development of the outflow tract myocardium. The specific molecular mechanisms by which this happens, while likely complex, remain to be elucidated and deserve additional research efforts. Interestingly, however, our data points toward the embryonic activation of the RAS pathway, known to be active in cardiac dysfunction later in life, as a possible mechanism inducing myocardial reorganization and cardiac defects in response to increase hemodynamic load. This study indicates that normal myocardial and mitochondrial reorganization is one of the initial processes affected by increased hemodynamic load, which induces a detrimental cascade of events that eventually leads to cardiac defects.

\section{AUTHOR CONTRIBUTIONS}

MM coordinated the study, carried out a large portion of the data collection and data analysis, and drafted the manuscript; CL carried out the work done in the Multiscale Microscopy Core in the OHSU Center for Spatial Systems Biomedicine and contributed to the manuscript; LD carried out the work and data analysis done in the OHSU Proteomics Core and contributed to the manuscript; AM helped with IPA analysis, SR conceived of the study and helped draft the manuscript.

\section{FUNDING}

This work was funded by a grant from US National Institutes of Health, NIH R01HL094570, and internal OHSU Center for Spatial Systems Biomedicine award. The proteomics work was also partially supported by an internal OHSU School of Medicine Faculty Innovation Fund award, and NIH grants P30EY01572, P30CA069533, and S10OD012246.

\section{ACKNOWLEDGMENTS}

Electron microscopy was performed at the Multi-scale Microscopy Core (MMC) with technical support from the OHSU-FEI Living Lab and the OHSU Center for Spatial Systems Biomedicine. Proteomic mass-spectrometry experiments were performed at the OHSU Proteomic Shared Resource with the assistance of John Klimek, Ashok Ready, and Phillip Wilmarth.

\section{SUPPLEMENTARY MATERIAL}

The Supplementary Material for this article can be found online at: http://journal.frontiersin.org/article/10.3389/fphys. 2017.00631/full\#supplementary-material 


\section{REFERENCES}

Bai, F., Wang, L., and Kawai, M. (2013). A study of tropomyosin's role in cardiac function and disease using thin-filament reconstituted myocardium. J. Muscle Res. Cell Motil. 34, 295-310. doi: 10.1007/s10974-013-9343-z

Beinlich, C. J., and Morgan, H. E. (1993). Control of growth in neonatal pig hearts. Mol. Cell. Biochem. 119, 3-9. doi: 10.1007/BF00926846

Beinlich, C. J., Rissinger, C. J., and Morgan, H. E. (1995). Mechanisms of rapid growth in the neonatal pig heart. J. Mol. Cell. Cardiol. 27, 273-281. doi: $10.1016 / \mathrm{S} 0022-2828(08) 80026-0$

Boengler, K., Heusch, G., and Schulz, R. (2011). Nuclear-encoded mitochondrial proteins and their role in cardioprotection. Biochim. Biophys. Acta 1813, 1286-1294. doi: 10.1016/j.bbamcr.2011.01.009

Brook, W. H., Connell, S., Cannata, J., Maloney, J. E., and Walker, A. M. (1983). Ultrastructure of the myocardium during development from early fetal life to adult life in sheep. J. Anat. 137(Pt. 4), 729-741.

Chen, H., Detmer, S. A., Ewald, A. J., Griffin, E. E., Fraser, S. E., and Chan, D. C. (2003). Mitofusins Mfn1 and Mfn2 coordinately regulate mitochondrial fusion and are essential for embryonic development. J. Cell Biol. 160, 189-200. doi: $10.1083 /$ jcb.200211046

Chung, S., Dzeja, P. P., Faustino, R. S., Perez-Terzic, C., Behfar, A., and Terzic, A. (2007). Mitochondrial oxidative metabolism is required for the cardiac differentiation of stem cells. Nat. Clin. Pract. Cardiovasc. Med. 4, S60-S67. doi: 10.1038/ncpcardio0766

Clark, E. B., and Rosenquist, G. C. (1978). Spectrum of cardiovascular anomalies following cardiac loop constriction in the chick embryo. Birth Defects Orig. Artic. Ser. 14, 431-442.

Clark, E. B., Hu, N., Frommelt, P., Vandekieft, G. K., Dummett, J. L., and Tomanek, R. J. (1989). Effect of increased pressure on ventricular growth in stage 21 chick embryos. Am. J. Physiol. Heart Circ. Physiol. 257(1 Pt. 2), H55-H61.

Culver, J. C., and Dickinson, M. E. (2010). The effects of hemodynamic force on embryonic development. Microcirculation 17, 164-178. doi: $10.1111 / j .1549-8719.2010 .00025 . x$

Damon, B. J., Remond, M. C., Bigelow, M. R., Trusk, T. C., Xie, W., Perucchio, R., et al. (2009). Patterns of muscular strain in the embryonic heart wall. Dev. Dyn. 238, 1535-1546. doi: 10.1002/dvdy.21958

Das, S., Steenbergen, C., and Murphy, E. (2012). Does the voltage dependent anion channel modulate cardiac ischemia-reperfusion injury? Biochim. Biophys. Acta 1818, 1451-1456. doi: 10.1016/j.bbamem.2011.11.008

Davies, P. F. (1995). Flow-mediated endothelial mechanotransduction. Physiol. Rev. 75, 519-560.

England, J., and Loughna, S. (2013). Heavy and light roles: myosin in the morphogenesis of the heart. Cell. Mol. Life Sci. 70, 1221-1239. doi: $10.1007 / \mathrm{s} 00018-012-1131-1$

Fields, S. D., Conrad, M. N., and Clarke, M. (1998). The S. cerevisiae CLU1 and D. discoideum cluA genes are functional homologues that influence mitochondrial morphology and distribution. J. Cell Sci. 111(Pt. 12), 1717-1727.

Fischman, D. A. (1967). An electron microscope study of myofibril formation in embryonic chick skeletal muscle. J. Cell Biol. 32, 557-575. doi: $10.1083 /$ jcb.32.3.557

Gao, J., Schatton, D., Martinelli, P., Hansen, H., Pla-Martin, D., Barth, E., et al. (2014). CLUH regulates mitochondrial biogenesis by binding mRNAs of nuclear-encoded mitochondrial proteins. J. Cell Biol. 207, 213-223. doi: $10.1083 /$ jcb. 201403129

Hale, D. E., Batshaw, M. L., Coates, P. M., Frerman, F. E., Goodman, S. I., Singh, I., et al. (1985). Long-chain acyl coenzyme a dehydrogenase deficiency: an inherited cause of nonketotic hypoglycemia. Pediatr. Res. 19, 666-671. doi: 10.1203/00006450-198507000-00006

Hamburger, V., and Hamilton, H. L. (1992). A series of normal stages in the development of the chick embryo. 1951. Dev. Dyn. 195, 231-272. doi: 10.1002/aja.1001950404

Hogers, B., DeRuiter, M. C., Gittenberger-de Groot, A. C., and Poelmann, R. E. (1997). Unilateral vitelline vein ligation alters intracardiac blood flow patterns and morphogenesis in the chick embryo. Circ. Res. 80, 473-481. doi: 10.1161/01.RES.80.4.473

Hogers, B., DeRuiter, M. C., Gittenberger-de Groot, A. C., and Poelmann, R. E. (1999). Extraembryonic venous obstructions lead to cardiovascular malformations and can be embryolethal. Cardiovasc. Res. 41, 87-99. doi: 10.1016/S0008-6363(98)00218-1

Hu, N., Christensen, D. A., Agrawal, A. K., Beaumont, C., Clark, E. B., and Hawkins, J. A. (2009). Dependence of aortic arch morphogenesis on intracardiac blood flow in the left atrial ligated chick embryo. Anat. Rec. 292, 652-660. doi: 10.1002/ar.20885

Hu, Y., Suarez, J., Fricovsky, E., Wang, H., Scott, B. T., Trauger, S. A., et al. (2009). Increased enzymatic O-GlcNAcylation of mitochondrial proteins impairs mitochondrial function in cardiac myocytes exposed to high glucose. J. Biol. Chem. 284, 547-555. doi: 10.1074/jbc.M808518200

Hüttemann, M., Klewer, S., Lee, I., Pecinova, A., Pecina, P., Liu, J., et al. (2012). Mice deleted for heart-type cytochrome c oxidase subunit 7al develop dilated cardiomyopathy. Mitochondrion 12, 294-304. doi: 10.1016/j.mito.2011.11.002

Knight, R. A., Scarabelli, T. M., and Stephanou, A. (2012). STAT transcription in the ischemic heart. JAKSTAT 1, 111-117. doi: 10.4161/jkst.20078

Liu, A., Yin, X., Shi, L., Li, P., Thornburg, K. L., Wang, R., et al. (2012). Biomechanics of the chick embryonic heart outflow tract at HH18 using 4D optical coherence tomography imaging and computational modeling. PLoS ONE 7:e40869. doi: 10.1371/journal.pone.0040869

Ma, Z., Liu, A., Yin, X., Troyer, A., Thornburg, K., Wang, R. K., et al. (2010). Measurement of absolute blood flow velocity in outflow tract of HH18 chicken embryo based on $4 \mathrm{D}$ reconstruction using spectral domain optical coherence tomography. Biomed. Opt. Express 1, 798-811. doi: 10.1364/BOE.1.000798

Manasek, F. J. (1970). Histogenesis of the embryonic myocardium. Am. J. Cardiol. 25, 149-168. doi: 10.1016/0002-9149(70)90576-X

McQuinn, T. C., Bratoeva, M., Dealmeida, A., Remond, M., Thompson, R. P., and Sedmera, D. (2007). High-frequency ultrasonographic imaging of avian cardiovascular development. Dev. Dyn. 236, 3503-3513. doi: 10.1002/dvdy.21357

Midgett, M., and Rugonyi, S. (2014). Congenital heart malformations induced by hemodynamic altering surgical interventions. Front. Physiol. 5:287. doi: $10.3389 /$ fphys.2014.00287

Midgett, M., Goenezen, S., and Rugonyi, S. (2014). Blood flow dynamics reflect degree of outflow tract banding in Hamburger-Hamilton stage 18 chicken embryos. J. R. Soc. Interface 11:20140643. doi: 10.1098/rsif.2014.0643

Midgett, M., López, C. S., David, L., Maloyan, A., and Rugonyi, S. (2017a). Increased hemodynamic load in early embryonic stages alters endocardial to mesenchymal transition. Front. Physiol. 8:56. doi: 10.3389/fphys.2017.00056

Midgett, M., Thornburg, K. L., and Rugonyi, S. (2017b). Blood flow patterns underlie developmental heart defects. Am. J. Physiol. Heart Circ. Physiol. 312, H632-H642. doi: 10.1152/ajpheart.00641.2016

Pappas, C. T., Mayfield, R. M., Henderson, C., Jamilpour, N., Cover, C., Hernandez, Z., et al. (2015). Knockout of Lmod2 results in shorter thin filaments followed by dilated cardiomyopathy and juvenile lethality. Proc. Natl. Acad. Sci. U.S.A. 112, 13573-13578. doi: 10.1073/pnas.1508273112

Pohjoismäki, J. L. O., Boettger, T., Liu, Z., Goffart, S., Szibor, M., and Braun, T. (2012). Oxidative stress during mitochondrial biogenesis compromises mtDNA integrity in growing hearts and induces a global DNA repair response. Nucleic Acids Res. 40, 6595-6607. doi: 10.1093/nar/gks301

Price, R. L., Carver, W., Simpson, D. G., Fu, L., Zhao, J., Borg, T. K., et al. (1997). The effects of angiotensin II and specific angiotensin receptor blockers on embryonic cardiac development and looping patterns. Dev. Biol. 192, 572-584. doi: 10.1006/dbio.1997.8764

Raeker, M. Ö., Shavit, J. A., Dowling, J. J., Michele, D. E., and Russell, M. W. (2014). Membrane-myofibril cross-talk in myofibrillogenesis and in muscular dystrophy pathogenesis: lessons from the zebrafish. Front. Physiol. 5:14. doi: 10.3389/fphys.2014.00014

Rai, R., Wong, C. C. L., Xu, T., Leu, N. A., Dong, D. W., Guo, C., et al. (2008). Arginyltransferase regulates alpha cardiac actin function, myofibril formation and contractility during heart development. Development 135, 3881-3889. doi: $10.1242 /$ dev. 022723

Rajan, S., Jagatheesan, G., Karam, C. N., Alves, M. L., Bodi, I., Schwartz, A., et al. (2010). Molecular and functional characterization of a novel cardiac-specific human tropomyosin isoform. Circulation 121, 410-418. doi: 10.1161/CIRCULATIONAHA.109.889725

Rennie, M. Y., Gahan, C. G., Lopez, C. S., Thornburg, K. L., and Rugonyi, S. (2014). 3D imaging of the early embryonic chicken heart with focused 
ion beam scanning electron microscopy. Microsc. Microanal. 20, 1111-1119. doi: $10.1017 / S 1431927614000828$

Roberts, D. E., Hersh, L. T., and Scher, A. M. (1979). Influence of cardiac fiber orientation on wavefront voltage, conduction velocity, and tissue resistivity in the dog. Circ. Res. 44, 701-712. doi: 10.1161/01.RES.44.5.701

Robinson, M. D., McCarthy, D. J., and Smyth, G. K. (2010). edgeR: a Bioconductor package for differential expression analysis of digital gene expression data. Bioinformatics 26, 139-140. doi: 10.1093/bioinformatics/ btp616

Rugonyi, S., Shaut, C., Liu, A., Thornburg, K., and Wang, R. K. (2008). Changes in wall motion and blood flow in the outflow tract of chick embryonic hearts observed with optical coherence tomography after outflow tract banding and vitelline-vein ligation. Phys. Med. Biol. 53, 5077-5091. doi: 10.1088/0031-9155/53/18/015

Sanger, J. W., Kang, S., Siebrands, C. C., Freeman, N., Du, A., Wang, J., et al. (2005). How to build a myofibril. J. Muscle Res. Cell Motil. 26, 343-354. doi: 10.1007/s10974-005-9016-7

Satou, R., and Gonzalez-Villalobos, R. A. (2012). JAK-STAT and the reninangiotensin system: the role of the JAK-STAT pathway in blood pressure and intrarenal renin-angiotensin system regulation. JAKSTAT 1, 250-256. doi: $10.4161 /$ jkst.22729

Schmieder, R. E., Hilgers, K. F., Schlaich, M. P., and Schmidt, B. M. W. (2007). Renin-angiotensin system and cardiovascular risk. Lancet 369, 1208-1219. doi: 10.1016/S0140-6736(07)60242-6

Sedmera, D., Pexieder, T., Rychterova, V., Hu, N., and Clark, E. B. (1999). Remodeling of chick embryonic ventricular myoarchitecture under experimentally changed loading conditions. Anat. Rec. 254, 238-252. doi: 10. 1002/(SICI)1097-0185(19990201)254:2<238::AID-AR10>3.0.CO;2-V

Shi, L., Goenezen, S., Haller, S., Hinds, M. T., Thornburg, K. L., and Rugonyi, S. (2013). Alterations in pulse wave propagation reflect the degree of outflow tract banding in HH18 chicken embryos. Am. J. Physiol. Heart Circ. Physiol. 305, H386-H396. doi: 10.1152/ajpheart.00100.2013

Smolich, J. J. (1995). Ultrastructural and functional features of the developing mammalian heart: a brief overview. Reprod. Fertil. Dev. 7, 451-461. doi: 10.1071/RD9950451

Sokol, A. M., Sztolsztener, M. E., Wasilewski, M., Heinz, E., and Chacinska, A. (2014). Mitochondrial protein translocases for survival and wellbeing. FEBS Lett. 588, 2484-2495. doi: 10.1016/j.febslet.2014.05.028

Sylvester, J. E., Fischel-Ghodsian, N., Mougey, E. B., and Orbrien, T. W. (2004). Mitochondrial ribosomal proteins: candidate genes for mitochondrial disease. Genet. Med. 6, 73-80. doi: 10.1097/01.GIM.0000117333.21213.17
Szczepanek, K., Chen, Q., Derecka, M., Salloum, F. N., Zhang, Q., Szelag, M., et al. (2011). Mitochondrial-targeted signal transducer and activator of transcription 3 (stat3) protects against ischemia-induced changes in the electron transport chain and the generation of reactive oxygen species. J. Biol. Chem. 286, 29610-29620. doi: 10.1074/jbc.M111.226209

Te Riet, L., van Esch, J. H. M., Roks, A. J. M., van den Meiracker, A. H., and Danser, A. H. J. (2015). Hypertension. Renin-angiotensin-aldosterone system alterations 116, 960-975. doi: 10.1161/CIRCRESAHA.116.303587

Tobita, K., Schroder, E. A., Tinney, J. P., Garrison, J. B., and Keller, B. B. (2002). Regional passive ventricular stress-strain relations during development of altered loads in chick embryo. Am. J. Physiol. Heart Circ. Physiol. 282, H2386-H2396. doi: 10.1152/ajpheart.00879.2001

Tsukada, T., Pappas, C. T., Moroz, N., Antin, P. B., Kostyukova, A. S., and Gregorio, C. C. (2010). Leiomodin-2 is an antagonist of tropomodulin-1 at the pointed end of the thin filaments in cardiac muscle. J. Cell Sci. 123, 3136-3145. doi: $10.1242 /$ jcs. 071837

Vendelin, M., Beraud, N., Guerrero, K., Andrienko, T., Kuznetsov, A. V., Olivares, J., et al. (2005). Mitochondrial regular arrangement in muscle cells: a "crystal-like" pattern. Am. J. Physiol. Cell Physiol. 288, C757-767. doi: 10.1152/ajpcell.00281.2004

Wainrach, S., and Sotelo, J. R. (1961). Electron microscope study of the developing chick embryo heart. Z. Zellforsch. Mikrosk. Anat. 55, 622-634. doi: $10.1007 / \mathrm{BF} 00384502$

Walker, J. E., and Runswick, M. J. (1993). The mitochondrial transport protein superfamily. J. Bioenerg. Biomembr. 25, 435-446. doi: 10.1007/BF01108401

Zhu, Q., Hulen, D., Liu, T., and Clarke, M. (1997). The cluA(-) mutant of Dictyostelium identifies a novel class of proteins required for dispersion of mitochondria. Proc. Natl. Acad. Sci. U.S.A. 94, 7308-7313. doi: $10.1073 /$ pnas.94.14.7308

Conflict of Interest Statement: The authors declare that the research was conducted in the absence of any commercial or financial relationships that could be construed as a potential conflict of interest.

Copyright @ 2017 Midgett, López, David, Maloyan and Rugonyi. This is an openaccess article distributed under the terms of the Creative Commons Attribution License (CC BY). The use, distribution or reproduction in other forums is permitted, provided the original author(s) or licensor are credited and that the original publication in this journal is cited, in accordance with accepted academic practice. No use, distribution or reproduction is permitted which does not comply with these terms. 